\title{
Proceedings of the 140th Semon Club,19 November 2010, ENT Department, Guy's and St Thomas' NHS Foundation Trust, London, UK
}

\author{
Chairman: Miss Elfy B Chevretton, Guy's and St Thomas' NHS Foundation Trust \\ Secretary: Mr Sherif Haikel, Guy's and St Thomas' NHS Foundation Trust \\ Invited panel for pathology: Professor Leslie Michaels and Dr Ann Sandison, University College London \\ Invited panel for radiology: Dr Steve Connor and Dr Ata Siddiqui, Guy's and St Thomas NHS Foundation Trust \\ Professor Michaels awarded a prize for the best presentation of the meeting to Dr Robert Nash ('Acute onset of alien limb \\ syndrome in a 27-year-old')
}

\author{
Otology and skull base session \\ Chairman: Mr Alec Fitzgerald O’Connor
}

An unusual and complex case of stapedial fracture

S Yalamanchili, S Rana, R Quiney

From The Royal Free Hospital, London

\section{Background}

Multiple stapedial fractures with preservation of other ossicles are unusual following penetrating ear injury. We present such a case, which was also, unusually, complicated by post-operative pneumovestibule.

\section{Case report}

A 39-year-old piano tuner presented with conductive hearing loss, having introduced the arm of her spectacles into her left ear.

Initial computed tomography (CT) showed no ossicular disruption. However, on exploratory tympanotomy a dislocated incudostapedial joint and fractured anterior stapes crus were repaired.

Hearing loss continued, and repeated exploration showed anterior and posterior stapes crura fractures. While removing the stapes suprastructure, the entire footplate was inadvertently lifted out due to a connecting adhesion and probable weakened annular ligament. A stapes prosthesis was inserted and fat packed around the oval window.

Post-operatively, the patient experienced tinnitus and paralytic nystagmus, and audiology suggested a 'dead ear'. Following treatment with steroids and antibiotics, audiometry still showed a marked conductive hearing loss.

Computed tomography demonstrated pneumovestibule, later successfully treated with saline to fill the vestibule and a fat and vein graft to seal the defect. Subsequently, the patient's hearing returned to normal.

\section{Discussion}

Dr Connor explained the difficulty in confirming stapes fractures on CT. Mr Quiney, who was not at the meeting, subsequently agreed that limited CT resolution had prevented the identification of abnormalities in this case.

A photograph of the removed stapes, apparently intact, raised questions regarding the necessity of stapedectomy, but Mr Quiney subsequently clarified that the stapes had been removed in fragments.

Mr Fitzgerald O'Connor asked whether gaps in the graft were noted intra-operatively. Mr Quiney subsequently confirmed that they had been, and suggested that repetitive post-operative vomiting may have caused fat displacement resulting in pneumovestibule.

\section{Lessons learnt}

Be cautious when encountering stapes fractures: the annular ligament may be tenuous.

Doctor my ear is leaking: what have you done?

S Patil, G Kansal, M Yung

From Ipswich Hospital

Introduction

Cerebrospinal fluid otorrhoea is rare after grommet insertion.

\section{Case report}

A 64-year-old man presented with left hearing loss over the previous few months. Audiology showed a mixed left hearing loss with a flat tympanogram. The patient underwent a left myringotomy and grommet insertion under local anaesthetic, resulting in a gush of clear fluid which subsequently tested positive for beta 2 transferrin. The grommet was removed after 48 hours as clear otorrhoea continued, and the patient was discharged on antibiotic eardrops. The patient represented to the accident and emergency department four days later with signs of meningitis, which was confirmed by lumbar puncture.

Radiology

Magnetic resonance imaging scans of the internal auditory meati were normal. A computed tomography (CT) scan of the mastoids showed poor pneumatisation on the left side, and soft tissue or fluid extending into the middle ear, with the suggestion of a defect in the left tegmen tympani.

\section{Management}

The meningitis was treated with appropriate antibiotics. The patient was referred to Addenbrooke's Hospital, where the tegmen defect was closed with a cartilage graft via a transmastoid approach. 
Conclusion and lessons learnt

Dr Connor commented that up to a third of normal patients have a very thin or absent tegmen on CT scan. Infratemporal lobe changes were suggestive of a previous contusion, and thus a traumatic aetiology of the tegmen defect was probable. Mr Fitzgerald O'Connor said he probably would not have inserted a grommet in this patient. Professor Howard reminded the audience of the other dangerous structures that may be encountered during myringotomy, such as a high jugular bulb and a misplaced carotid artery.

Primary intraosseus meningioma of the temporal bone

A Koukkoullis, C King, M Lyons

From Lister Hospital, Stevenage

\section{Introduction}

Primary extradural meningiomas are rare lesions which constitute less than 2 per cent of all meningiomas. Fourteen per cent of primary extradural meningiomas are intraosseous, and these lesions are often confused with primary bone tumours of the skull.

\section{Case report}

An 83-year-old woman presented with a left inferior temporal quadrant visual field defect and bilateral pale optic discs. There was a large, visible mass in the left temporal region, present for over two years and slowly increasing in size. She was otherwise asymptomatic.

\section{Radiological findings}

Magnetic resonance imaging and computed tomography demonstrated a solitary, expansile, densely sclerotic lesion in the left temporal bone. There was smooth enhancement of the underlying dura but no evidence of invasion. The differential diagnosis included osteosarcoma, haemangioma and meningioma.

\section{Histological findings}

Histological analysis showed bone fragments infiltrated by a tumour composed of short fascicles and whorl-like clusters of small, ovoid cells consistent with meningioma. This was supported by immunohistochemical analysis.

\section{Management}

The patient was referred to Addenbrooke's Hospital for audiological follow up without surgical intervention.

\section{Discussion}

Dr Sandison suggested that calvarian primary extradural meningiomas could be the result of thin strands of dura, not visualised with current imaging, invading through the cortex into the diploic space. Professor Howard emphasised that primary extradural meningiomas can be found in younger patients. Although primary extradural meningiomas can occur anywhere in the body, they are often diagnosed by ENT surgeons. Extensive surgical resection is required due to the finger-like projections of meningioma into the bone, which are often connected to the dura. In young patients, early identification and excision is imperative, as with time these tumours can grow to an inoperable size, and are five times more likely to become malignant. However, elderly patients should only be treated if symptomatic and radiological follow up is the treatment of choice.
An unusual cause of middle-ear effusion in an adult

C Abbassi, F Sipaul, R Youngs

From Gloucestershire Royal Hospital, Gloucester

\section{Background}

A bony defect of the tegmen tympani leading to cerebrospinal fluid (CSF) leakage is a rare cause of middle-ear effusion.

\section{Case report}

A 64-year-old man presented with a five-year history of mild hearing loss in his left ear, with no other ear symptoms. This was preceded by admission to the intensive care ward for exacerbation of severe chronic obstructive pulmonary disease. There was no prior history of head trauma. A diagnosis of middle-ear effusion was made based on audiometric findings, and a grommet was inserted. Following this, the patient's left ear continued to discharge clear fluid. This was initially treated with Gentisone-HC drops. When the discharge failed to resolve, the fluid was sent for laboratory analysis, which confirmed that it was CSF.

\section{Radiological findings}

A computed tomography (CT) scan of the left petrous bone showed a breach in the tegmen and a soft tissue density in the mastoid air cells extending to the majority of the middle-ear cavity, which might be cholesteatoma.

\section{Management}

A left middle fossa approach was used to surgically close the CSF fistula.

\section{Discussion}

Cerebrospinal fluid otorrhoea without apparent cause poses a diagnostic difficulty. A strong degree of suspicion and a CT scan are required. Mr Fitzgerald O'Connor held the opinion that a magnetic resonance imaging scan was warranted in this case before any surgical intervention. The tegmen defect size was unknown in this case; however, a large temporalis fascia graft was needed for closure. Professor Howard agreed that a middle fossa approach was preferable for large defects, but that confirmation with further imaging was necessary due to the serious complications associated with this procedure.

\section{Osteoradionecrosis of the temporal bone}

\section{R Sharma, R Obholzer, M Gleeson}

From Guy's Hospital, London

\section{Introduction}

We present the case of a 67-year-old woman who developed left-sided otalgia and otorrhoea following extensive radiotherapy to the parotid bed.

\section{Case report}

The patient had had a left pleomorphic adenoma incompletely resected in 1966, aged 23 years. The tumour had recurred periodically, and after the fourth resection, 33 years later, the parotid bed had been irradiated with 50 Gy in 25 fractions. Carcinoma ex pleomorphic adenoma had been diagnosed eight years later, and treated with further resection and irradiation with $47 \mathrm{~Gy}$ in 23 fractions. The patient had developed progressive otalgia and otorrhoea following the second course of radiotherapy. Examination revealed exposed, infected bone in the external auditory 
canal, leading to a diagnosis of osteoradionecrosis of the temporal bone.

\section{Radiology}

Computed tomography appearances were compatible with osteoradionecrosis of the temporal bone, with inflammation extending to the petrous apex.

\section{Management}

The patient underwent left lateral petrosectomy with a blind sac closure of the external auditory canal. A vascularised temporo-parietal fascial flap, raised on the branches of the superficial temporal artery, was used to reconstruct the surgical defect and to introduce a viable blood supply to relatively avascular tissue. Subsequent venous congestion of the pinna was treated with leech therapy.

\section{Conclusion and lessons learnt}

This case highlights the risk of osteoradionecrosis of the temporal bone after radiotherapy to the parotid gland, the importance of addressing the cardinal symptom of otalgia, and the surgical options for treatment. During the meeting, Professor Howard also highlighted the potential role of hyperbaric oxygen therapy in the medical management of this condition.

\section{Rhinology session}

Chairman: Mr David Roberts

\section{A rare cause of unilateral nasal obstruction in an adult}

K Hanks, F Sipaul, R P Youngs

From Gloucestershire Royal Hospital, Gloucester

\section{Case report}

Unilateral nasal lesions require histological evaluation to exclude malignancy, along with imaging to determine the full extent. Management is dependent upon results, and misdiagnosis can have serious implications. A 44-year-old man presented with a left-sided nasal polyp. A biopsy was taken in the clinic.

\section{Radiological findings}

Computed tomography of the sinuses showed a solitary, polypoid lesion in the left nasal cavity, with no evidence of bony erosion.

\section{Histological findings}

The appearance of the clinic biopsy was consistent with an inverted sinonasal papilloma.

\section{Management}

A pedunculated tumour originating from a vascular pedicle on the septum and sphenoid was resected. Excessive bleeding was noted.

Histological analysis of the resected specimen showed a glomangiopericytoma.

The multidisciplinary team felt that although the tumour was benign, complete excision and follow up with imaging was advisable due to its potentially aggressive nature.

\section{Conclusion and lesson learnt}

Following resection, the initial biopsy was reviewed and found to have the same histological features as the subsequently resected lesion. The initial misdiagnosis led to the patient undergoing a surgical procedure, with its associated risks.
At the meeting, Professor Howard stated that large, unilateral nasal lesions should not be biopsied in the clinic but rather examined under anaesthesia and a biopsy taken from a non-air-exposed surface. He also added that a magnetic resonance imaging scan would have revealed the vascular nature of the lesion prior to the resection. Dr Sanderson agreed on the importance of ensuring that the biopsy was representative of the lesion as a whole.

Don't sue the surgeon: remineralisation of the skull base or a defect that never existed?

S Dhillon, D Roberts, C Hopkins

From Guy's Hospital, London

\section{Introduction}

Cerebrospinal fluid (CSF) rhinorrhoea resulting from an anterior skull base defect is a rare but major complication of functional endoscopic sinus surgery (FESS), with an estimated incidence of 0.04 per cent.

\section{Case report}

A 56-year-old woman was referred having previously undergone FESS, following which she developed meningitis. She subsequently reported daily headaches and persistent rhinorrhoea. A computed tomography (CT) scan demonstrated a significant defect of the anterior skull base, reported by two independent radiologists.

Exploration of the skull base six weeks after initial surgery found that not only was the skull base intact but the overlying mucosa appeared undisturbed. No CSF leakage was identified intra-operatively.

The scans were reviewed and found to be reconstructed coronal images. Repeated CT performed three weeks postoperatively demonstrated a complete bony plate, and the patient no longer reported any symptoms.

\section{Discussion}

Computed tomography is the mainstay of CSF rhinorrhoea investigation due to its ability to identify osseous defects. In this case, we speculated that the defect was a virtual artefact of CT image reconstruction. We cannot exclude the alternative possibility of remineralisation, but given the time-frame this appears unlikely, as the average reported length of time required is six to nine months. Dr Connor noted the absence of a lateral cribriform plate on the right side. Professor Michaels highlighted the fact that cortical bone appears abnormal following remineralisation, hence supporting the theory that the apparent defect did not in fact exist. Professor Howard agreed, adding that the cortical bone of the skull base does not regenerate.

\section{Conclusion}

This case emphasises the importance of caution when interpreting reconstructed images of the skull base. If doubt exists, high resolution, directly acquired CT should more accurately confirm continuity of the bony margins.

Obstructive and erosive unilateral nasal mass in a patient with metastatic ovarian adenocarcinoma

\section{S N Ifeacho, J Ho, S A Hannan}

From The Royal Free and The Royal National Throat, Nose and Ear Hospitals, London 
Introduction

Primary sinonasal tumours account for 0.5 per cent of all malignancies. Metastatic tumours are even rarer.

\section{Case report}

A 52-year-old woman presented with left-sided nasal blockage and a past medical history of metastatic ovarian adenocarcinoma. Clinical examination revealed total obstruction of the left nasal cavity by a friable, polypoid lesion.

\section{Radiology}

Computed tomography demonstrated a mass filling the left nasal cavity, ethmoid and sphenoid sinuses and eroding the posterior half of the cribriform plate, lamina papyracea and medial aspect of the fovea ethmoidalis.

Magnetic resonance imaging with gadolinium showed a homogeneously enhancing lesion crossing the midline into the right superior nasal recess. The most posterior part of the lesion extended into the left eustachian tube.

\section{Histology}

Tissue sections showed mucosal infiltration by a necrotic, high grade, poorly differentiated squamous cell carcinoma with acantholytic features. Immunohistochemical analysis was positive for cancer antigen 125 and tumour protein 63 (suggesting squamous differentiation).

\section{Management}

The patient underwent endoscopic resection of the lesion, and at the time of writing remained under the surveillance of both the head and neck and the gynaecology and oncology multidisciplinary teams.

\section{Conclusion}

Even in the presence of a history of active, metastatic cancer, sinonasal tumours are more likely to be primary rather than secondary lesions. Clinical differentiation between the two, by radiological and histological analysis, is essential for diagnosis. This case highlights the importance of a multidisciplinary approach when managing such patients.

Acute onset of alien limb syndrome in a 27-year-old

R Nash, N Kitchen

From The National Hospital for Neurology and Neurosurgery, London

\section{Introduction}

It is rare to diagnose skull base pathology in patients presenting with unusual neurological symptoms such as alien limb syndrome. We present such a case and review the literature on spontaneous pneumocephalus.

\section{Case report}

A 27-year-old woman presented to the neurology service with a three-day history of her left arm performing movements without voluntary control, together with decreased coordination in her left leg. She was otherwise well, with a past medical history of right-sided sinus surgery aged 11 years for recurrent sinusitis.

\section{Radiological findings}

A magnetic resonance imaging scan of the brain demonstrated a large, gas-filled collection in the right frontal lobe. Computed tomography confirmed the presence of pneumocephalus, apparently originating from an enostosis and defect in the anterior skull base.

\section{Histological findings}

The appearance of the excised enostosis was in keeping with normal lamellar bone.

\section{Management}

The patient underwent craniotomy, with excision of the enostosis, aspiration of the pneumocephalus and repair of the cribiform plate. She made an excellent recovery and was discharged one week after presentation.

\section{Conclusion}

Spontaneous pneumocephalus is a rare disorder that arises as a consequence of barotrauma and skull base disorders. This case was unusual for the nature of its presentation. Professor Howard speculated that this may have been due to a subacute accumulation of intracranial air. A variety of approaches to management were discussed, dependent on the underlying aetiology, the patient's clinical state, and the expertise of the attending clinician.

Unilateral nasal blockage, rhinorrhoea and epiphora resistant to medication

\section{Rashid, J Chan, Y G Karagama}

From Tameside Hospital, Ashton-under-Lyne

\section{Introduction}

We present a case of a large, diffuse B-cell lymphoma of the maxillary sinus.

\section{Case report}

A 57-year-old woman presented with a four-month history of left-sided nasal blockage, rhinorrhoea, epiphora and leftsided facial puffiness. Examination revealed a diffuse swelling over the left maxillary antrum extending to the temporal region. There was no proptosis, and fibre-optic endoscopy showed medial displacement of the left lateral nasal wall. The patient also reported non-specific visual disturbances at presentation.

\section{Radiology}

Computed tomography (CT) showed a lobulated mass filling the left maxillary antrum and nasal fossa, with erosion of the medial maxillary wall and the alveolar margin of the maxillary bone. A magnetic resonance imaging (MRI) scan was also performed for staging, after histological confirmation of the diagnosis.

\section{Management}

Left-sided endoscopic debulking of the tumour with completion of the medial maxillectomy was performed under image guidance.

\section{Histology}

Biopsy confirmed the presence of a large, diffuse, high-grade B-cell lymphoma.

Chemotherapy has both a curative and palliative role in lymphoma cases, although radiotherapy is also advocated for periorbital disease.

\section{Conclusion}

The authors would advocate maintaining a low threshold of clinical suspicion for lymphoma in cases of unilateral nasal obstruction and presumed protracted sinusitis, with mandatory CT and MRI scanning. Mr Roberts said that he would have only biopsied the tumour, as opposed to debulking. Professor Howard agreed that both CT and MRI scans 
should be performed prior to any procedure for suspicious nasal lesions.

\section{Paediatric session \\ Chairman: Mr Gavin Morrison}

A complicated emergency: periorbital cellulitis in a child with craniofacial abnormalities

P Touska, S Gupta, P Chatrath

From Queen's Hospital, Romford

\section{Introduction}

Periorbital cellulitis is an uncommon but serious condition which is usually readily identified at presentation. We present a case in a child with Pfeiffer syndrome, a rare condition characterised by premature fusion of the cranial sutures and midface hypoplasia. The diagnostic challenges which arose in the context of this coexisting craniofacial syndrome are highlighted and discussed.

\section{Case}

A 14-year-old boy with Pfeiffer syndrome presented with a one-day history of progressively worsening headache, photophobia and ocular pain. On examination, he was febrile with right-sided proptosis and periorbital swelling.

\section{Radiology}

An urgent initial computed tomography scan confirmed periorbital cellulitis, but a significant, possibly infected, frontal collection was also visible. Subsequent magnetic resonance imaging revealed the nature of the collection: a large collection of cerebrospinal fluid (CSF) within the frontal bone, communicating with the ventricles.

\section{Management}

The patient was initially treated with broad-spectrum antibiotics. However, he deteriorated, developing acute hydrocephalus secondary to Streptococcus pneumoniae infection. The patient subsequently underwent a ventriculostomy and made an excellent post-operative recovery.

\section{Discussion}

Mr Morrison agreed with the decision to avoid drainage in the first instance and instead to arrange further imaging. Professor Howard added that to prevent future recurrence in this patient, neurosurgical repair of the CSF defects was likely to become unavoidable.

\section{Conclusion}

Ultimately, expeditious and complementary input from several specialities facilitated the appropriate management of a rapidly evolving and challenging case.

An unusual head and neck mass in a neonate

J E H M Bates, R P Exley, I A Bruce

From The Royal Manchester Children's Hospital

\section{Introduction}

Infantile tumours are a disparate group of neoplasms which may present a difficult problem for clinicians, with accurate histopathological diagnosis essential for treatment planning.
Case report

At birth, a female infant was noted to have a large, pigmented neck mass. This had not been identified at antenatal screening.

\section{Radiological findings}

On ultrasound scanning, areas of calcification were seen. Therefore, the lesion was thought to possibly represent a neuroblastoma. Computed tomography demonstrated a heterogeneous mass of soft tissue density, containing central, irregular calcification measuring $58 \times 43 \mathrm{~mm}$. At this stage, teratoma was included in the differential diagnosis.

\section{Histological findings}

On histological examination, the mass demonstrated a prominent, haemangiopericytoma-like vascular pattern with numerous thick-walled, branching vessels that had a stag horn configuration, with no evidence of atypia or pleomorphism. The vascular pattern and strong positivity for cluster of differentiation 34 glycoprotein lead to the diagnosis of infantile haemangiopericytoma.

\section{Management}

After discussion amongst the multidisciplinary team, the decision was made to attempt complete surgical excision of the mass. The mass was resected without complications, and a diagnosis of infantile haemangiopericytoma was subsequently reached. Those present at both the multidisciplinary team meeting and the Semon club meeting agreed that six-monthly follow up for five years was essential due to the borderline malignancy of the tumour.

\section{Conclusion and lessons learnt}

If rare childhood tumours of the head and neck are to be managed effectively, a multidisciplinary approach combining oncologists, surgeons and pathologists is imperative. This case illustrates the need for early multidisciplinary intervention, especially for complex infantile tumours of unknown diagnosis and borderline malignancy.

\section{Catastrophic complication of an upper respiratory} tract infection

M Kadr, H Burns, I Hore

From Guy's and St Thomas' NHS Trust, London

Retropharyngeal abscess is a well known complication of tonsillitis. However, skull base osteomyelitis with occlusion of the internal carotid and subsequent cerebrovascular accident has not previously been reported in the literature.

A four-year-old girl, previously fit and well, was transferred to the Evelina Children's Hospital with a history of fever, vomiting and neck stiffness. This followed a week's prodrome of febrile illness, pharyngitis and otitis media. Left-sided neurological signs, with a VIth cranial nerve palsy, then developed.

Magnetic resonance imaging showed a retropharyngeal abscess with extension of an infective process into the parapharyngeal space and central skull base. There was thrombophlebitis of the cavernous sinuses, with marked external compression of the internal carotid arteries and watershed distribution acute infarcts in the right cerebral hemisphere.

Drainage of the retropharyngeal abscess, sphenoidotomy and insertion of grommets was performed. Microbiological culture grew Pseudomonas aeruginosa, and treatment was commenced with gentamicin, Tazocin, metronidazole and anticoagulation. The patient improved on this regime; 
however, antibiotics were changed after three weeks due to a rash, with consequent deterioration and sepsis. The original antibiotics were reintroduced and antifungals added, which resulted in resolution of the sepsis.

At the time of writing, long term monitoring of the skull base osteomyelitis with positron emission tomography was planned, together with continuation of long term antibiotic and antifungal therapy.

This case demonstrates the potential complications of retropharyngeal abscess and the need for prolonged antimicrobial treatment.

Mr Morrison agreed that uncommon but serious infections should be treated aggressively, and that anticoagulation was recommended for vascular compromise.

\section{Significant progressive pathology presenting as a} neck mass

H Burns, M Kadr, I Hore

From Guy's and St Thomas' NHS Trust, London

Paediatric cervical lymphadenopathy is usually a benign presentation, but may occasionally represent more sinister pathology.

We present a case of a nine-year-old boy of African origin, who while receiving treatment for tuberculosis (TB) developed right-sided cervical lymphadenopathy. This increased in size as the patient's anti-TB regime continued. He was referred to the ENT department at St Thomas' Hospital six months after the nodes had first been noted, at which stage they measured $7 \times 3 \mathrm{~cm}$. The patient had already undergone fine needle aspiration cytology (FNAC) which showed features of a reactive node and no suggestion of ongoing TB or lymphoma. Ultrasound confirmed a matted collection of neck nodes. He was otherwise well.

Histological analysis of a subsequent neck node biopsy indicated the presence of nasopharyngeal carcinoma. Flexible nasendoscopy identified a mass in the nasopharynx. A computed tomography and positron emission tomography scan unfortunately demonstrated distant metastasis. Despite chemotherapy, his disease progressed and his care was transferred to the palliative care team.

This case illustrates the importance of excisional biopsy when FNAC is inconclusive or does not fit with the clinical presentation. Nasopharyngeal cancer, although rare in children, may be associated with Epstein-Barr virus. Immunosuppression, as during TB treatment, may predispose to malignant transformation. Although generally this malignancy responds well to chemotherapy, distant metastases carry a very poor prognosis.

Professor Michaels stressed to the audience the significance of ethnic variations in the prevalence of nasopharyngeal carcinoma, it being more common in northern African areas.

\section{A large parapharyngeal mass in a child}

J Magill, C Butler, G Sandhu

From Charing Cross Hospital, London

\section{Introduction}

We report an eight-year-old girl who presented with an unusual right parotid swelling. Histological examination demonstrated a cystic hygroma.
Case report

The patient presented with a four-month history of a painless right parotid swelling which was gradually increasing in size. Clinical examination revealed a right parotid swelling which was displacing the tonsil medially. The exact nature of this lesion was clinically indeterminate.

\section{Radiology}

Magnetic resonance imaging showed a large mass in the parapharyngeal space deviating down from the right tonsil towards the midline and causing the parotid to be displaced laterally. The radiological findings were consistent with a cystic mass.

\section{Management}

The patient underwent total excision of the parapharyngeal lesion. During the operation, it was noted that the mass had features of a cystic hygroma. This diagnosis was confirmed on histological examination.

\section{Conclusion and lessons learnt}

Cystic hygroma is a benign lymphatic lesion which commonly presents at birth and rarely over the age of two years. Diagnosis is usually clinically apparent but an atypical presentation may prove challenging. Surgery is considered the main treatment of choice, but proved to be a topic of debate during the Semon Club meeting. Mr Morrison said that he would have considered injecting sclerosants due to the difficulty of surgical excision, whilst Professor Howard said he would only inject unilocular lesions, since injecting multilocular lesions would make subsequent surgical excision very difficult.

\section{Head and neck session}

\section{Chairman: Mr Ricard Simo}

\section{A case of chronic sclerosing sialadenitis (Kuttner's tumour) involving all four major salivary glands}

\section{R Cetto, A Ayub, D W McPartlin \\ From Queen Elizabeth Hospital, King's Lynn}

Chronic sclerosing sialadenitis is a rare condition also known as Kuttner's tumour. Clinically, this condition appears as a firm mass found usually in one of the salivary glands and resembling a neoplasm. Histologically, it is characterised by progressive periductal sclerosis, dense lymphocytic infiltration with lymphoid follicle formation, acinar atrophy and fibrosis.

A 79-year-old man presented with a four-month history of a $2 \mathrm{~cm}$ swelling in his left submandibular gland, which was gradually increasing in size. Ultrasound revealed a lobulated, heterogeneous swelling measuring $13 \times 13 \times 15 \mathrm{~mm}$, consistent with a submandibular gland tumour. Ultrasoundguided fine needle aspiration cytology (FNAC) showed no evidence of neoplasia. The left submandibular gland was excised. Histological findings were consistent with Kuttner's tumour.

Nine months later, the patient returned to the clinic complaining of an asymptomatic, right submandibular swelling. On examination, there were also residual left submandibular and bilateral parotid swellings. Fine needle aspiration cytology did not reveal neoplasia.

This case is an example of Kuttner's tumour involving all major salivary glands. Kuttner's tumour creates an 
interesting diagnostic dilemma as, although it is a benign lesion, it resembles closely the clinical characteristics of malignancy. Furthermore, 50 per cent of tumours presenting in the submandibular gland are malignant, complicating the management of the condition even further. Kuttner's tumour has been sporadically reported since 1896, yet it is still under-diagnosed. Ultrasound-guided FNAC is useful in excluding malignancy. After diagnosis, conservative management is a good approach. Professor Howard commented that Kuttner's tumour is part of the spectrum of lymphoproliferative disease.

\section{An unusual cause of stridor}

N Hamilton, C Al Yaghchi, G Sandhu

From Charing Cross Hospital, London

\section{Introduction}

We present a 34-year-old man with treatment-resistant subglottic stenosis that proved to be a diagnostic challenge.

\section{Case report}

The patient presented with stridor, and investigation revealed a circumferential subglottic thickening. He underwent multiple microlaryngoscopy and laser procedures to treat the stenosis but the lesion recurred. Biopsies showed a chronic inflammatory process but autoimmune and viral screening were negative. He had no recent history of foreign travel. The patient received immunosuppressant therapy to treat a possible relapsing polychondritis. When he failed to respond, a laryngofissure with deep biopsies was performed.

\section{Radiological findings}

A computed tomography scan at presentation revealed a circumferential, subglottic soft tissue mass. Further imaging showed that the mass expanded into the glottis.

\section{Histological findings}

Deep subglottic biopsies revealed an intense chronic inflammatory cell infiltrate, including sheets of 'foamy' histiocytes consistent with Mikulicz cells. Bacilli present within these cells were demonstrated on Warthin-Starry silver tinctorial stain. These findings were consistent with infection by Klebsiella rhinoscleromatis.

\section{Management}

The patient was given a two-week course of intravenous ceftriaxone and ciprofloxacin followed by long-term oral ciprofloxacin. At follow up, the patient's airway had improved, and further biopsies are planned to determine the response to treatment.

\section{Conclusion and lessons learnt}

Rhinoscleroma is a chronic granulomatous condition that usually affects the nose. It is caused by infection with Klebsiella rhinoscleromatis and is endemic in certain regions but rare within Western Europe, with no case of laryngeal rhinoscleroma yet reported within the UK. This case highlights the importance of obtaining representative samples for histology, even in relatively inaccessible regions such as the subglottis.
An unusual thyroid mass

A Zeinati, T Odutoye

From St George's Hospital, London

We report the case of a previously well, 62-year-old woman who presented with a three-month history of a large, asymptomatic, left-sided neck mass. Physical examination demonstrated a palpable, firm mass in her left lower neck, between levels IV, VI and VII, which clinically appeared to be of thyroid origin.

Ultrasonography suggested a large, dominant nodule of the left thyroid lobe, whilst fine needle aspiration cytology (FNAC) supported a diagnosis of a non-Hodgkins peripheral T-cell lymphoma. A magnetic resonance imaging scan also supported the theory that this was a thyroid mass. Computed tomography scans of the thorax, abdomen and pelvis were otherwise normal. A decision was made to perform a diagnostic incisional biopsy of the mass. Histological analysis indicated a diagnosis of ectopic cervical thymoma. The patient subsequently underwent a left hemi-thyroidectomy and excision of a left-sided cervical thymoma, together with a left selective neck dissection (level VI nodes).

Ectopic cervical thymoma is a rare tumour. It is derived from thymic epithelium and consists of a mixture of epithelial and lymphoid cells. It is frequently misdiagnosed on FNAC. Although thymomas are commonly found in the anterior mediastinum, they can arise from rests of ectopic thymic tissue caused by defective migration of the embryonic thymus. Due to their anatomical location, they are commonly mistaken for thyroid or parathyroid lesions. When approaching a neck mass with conflicting radiological and pathological findings, it is very important to proceed cautiously using the full breadth of the investigative arsenal, prior to definitive treatment.

\section{Facing a diagnostic dilemma}

L Ferguson, R Hewitt, A Mace

From Charing Cross Hospital, London

\section{Introduction}

We present a case of a 44-year-old man presenting with extensive soft tissue destruction of the right cheek, which proved to be a diagnostic dilemma.

\section{Case report}

This man presented with a two-month history of a rapidly progressing, destructive lesion of the right cheek, which had started as an intra-oral lesion. Clinically, there was soft tissue loss on the right cheek, eroding into bone. On investigation, the patient was found to be human immunodeficiency virus positive.

\section{Radiology}

Computed tomography showed soft tissue loss and abnormal enhancement of the surrounding tissue contiguous with an area of bony destruction within the right maxilla. There was a soft tissue mass extending almost as far as the posterior margin of the vertical ramus of the mandible, inseparable from the masseter muscle. There was also a large, level I lymph node.

\section{Management}

Treatment consisted of surgical debridement and longterm intravenous antibiotics, followed by staged surgical reconstruction. 


\section{Histology}

Histological investigation demonstrated skin and subcutaneous tissue with extensive necrosis, ulceration, and acute and chronic inflammation. Numerous filamentous organisms were seen with Warthin-Starry stain, forming colonies on the surface and infiltrating through tissue.

\section{Conclusion}

The features were consistent with noma (also known as gangrenous stomatitis). Noma is extremely rare in the Caucasian population, and is generally regarded as a disease of children in sub-Saharan Africa.

We present this patient to the Semon club meeting to highlight the diagnostic challenges of this case, and to suggest that this disease be considered in the differential diagnosis of destructive facial lesions in the immunocompromised.

Not a simple laryngeal schwannoma!

C Al Yaghchi, I Amer, K Ghufoor

From St Bartholomew's Hospital, London

\section{Introduction}

Solitary fibrous tumour is a mesenchymal tumour of probable fibroplastic type. Laryngeal solitary fibrous tumours are very rare, with only a few cases in the medical literature; all were benign tumours with no sign of recurrence.

\section{Case report}

A 78-year-old woman first presented to our care in 1990 when her laryngeal tumour was excised surgically. It was diagnosed initially as a schwannoma. Over the next 17 years, she had three further, recurrent tumours excised. The last of these was confirmed to be a solitary fibrous tumour. To our best knowledge, this case is the first to show recurrence of a laryngeal solitary fibrous tumour with a potentially malignant nature.

\section{Radiological findings}

Magnetic resonance imaging showed two discrete masses in the right supraglottic area, almost completely occluding the airway.

\section{Histological findings}

Histological examination revealed a soft tissue neoplasm composed of spindle cells arranged in a haemangiopericytoma-like pattern. Immunohistochemical staining was positive for cluster of differentiation 34 glycoprotein. The lesion was considered malignant or 'at best potentially metastatic' due to its recurrence, cellularity, atypia and infiltrative nature.

\section{Management}

The patient underwent $\mathrm{CO}_{2}$ laser assisted endoscopic resection. Three separate lesions were excised in the lateral pharyngeal wall and the supraglottic area.

\section{Discussion}

Dr Sandison explained that solitary fibrous tumour of the larynx is extremely rare. These tumours can be difficult to diagnose histologically as they share some features with other fibromatous tumours. Professor Howard agreed that the best treatment for this patient was palliative $\mathrm{CO}_{2}$ laser resection, performed due to her comorbidities. 